\title{
A new coumarin from stem bark of Mesua hexapetala
}

\begin{abstract}
A new alkylated coumarin derivative, hexapetarin (1) along with three other xanthones, trapezifolixanthone (2), cudraxanthone G (3) and 1,3,7-trihydroxy-2,4-di (3-methyl-2butenyl)xanthone (4), and four common triterpenoids, friedelin (5), stigmasterol (6), betasitosterol (7) and gamma-sitosterol (8) were isolated from the stem bark of Mesua hexapetala (Clusiaceae), a plant, native to Malaysia. The structures of these compounds were elucidated and determined using spectroscopic techniques such as NMR and MS. Anti-inflammatory activity assay indicated hexapetarin (1) to possess moderate anti-inflammatory activity, while 1,3,7-trihydroxy-2,4-di (3-methyl-2-butenyl)xanthone (4) gave very good activity.
\end{abstract}

Keyword: Mesua hexapetala; Anti-inflammatory; Coumarin; Xanthone 